\title{
Cloaking and Holography Experiments Using Immersive Boundary Conditions
}

\author{
Nele Börsing $\oplus,{ }^{*}$ Theodor S. Becker®, Andrew Curtis $\odot,{ }^{\dagger}$ Dirk-Jan van Manen $\odot$, Thomas Haag, and \\ Johan O.A. Robertsson (1) \\ ETH Zürich, Institute of Geophysics, 8092 Zürich, Switzerland
}

(Received 13 March 2019; revised manuscript received 20 June 2019; published 6 August 2019)

\begin{abstract}
We report the experimental realization of real-time and broadband acoustic cloaking and holography by manipulating wave fields through their boundary conditions. The method of immersive boundary conditions (IBCs) enables us to virtually replace part of a physical experiment with a virtual computational environment. By introducing a source surface enclosing the virtual environment and calculating the source strengths in real time, the wave field in the physical experiment can be controlled such that incoming waves are suppressed and transmissions through the arbitrary virtual domain are radiated in a desired manner. A one-dimensional experiment shows that scattering objects can be made undetectable without prior knowledge about the incident wave field. Moreover, virtual objects or media can be emulated where they are not present - even objects or media exhibiting properties that do not occur in nature. Our results provide useful insights into how to implement IBCs for dynamic cloaking and holography, suggesting that higher-dimensional experiments are feasible.
\end{abstract}

DOI: 10.1103/PhysRevApplied.12.024011

\section{INTRODUCTION}

The work described here is motivated by the desire to fully control and manipulate wave fields through their acoustic boundary conditions in a physical wave-propagation experiment. By controlling boundary conditions, we wish to suppress forward and backward scattering from objects so that each object effectively becomes undetectable. We wish to place virtual (imagined) objects within a physical environment and vice versa such that physical waves pass from one to the other as if both environments were real.

The quest to control boundary conditions has received significant attention across disciplines, ranging from electromagnetics [1], through optics [2] and acoustics [3] to the education and entertainment industry [4,5] and approaches generally fall into two categories: passive and active methods. While passive methods are based on coating the boundary with materials that alter wave-propagation paths, active methods use sources to manipulate incoming and control outgoing waves.

\footnotetext{
*nele.boersing@erdw.ethz.ch

${ }^{\dagger}$ Also at School of GeoSciences, University of Edinburgh, Edinburgh, United Kingdom.

Published by the American Physical Society under the terms of the Creative Commons Attribution 4.0 International license. Further distribution of this work must maintain attribution to the author(s) and the published article's title, journal citation, and DOI.
}

Motivated by the development of electromagnetic metamaterials [6-9], passive methods to steer acoustic waves have gained much attention over the past decade. Theoretical and experimental advances in the design of acoustic metamaterials [3] have produced medium properties such as enhanced absorption, negative refraction, or extremely high anisotropy. Acoustic cloaking is one of the most widely studied innovations using metamaterials. Numerous invisibility devices have been proposed, with potential applications in the fields of noise reduction, architectural acoustics, civil engineering, acoustic imaging, and underwater acoustics [10-14].

Moreover, Refs. [15] and [16] have shown that metamaterials can also be designed to create acoustic illusions. Instead of only eliminating the scattering from an object, an "illusion cloak" modifies its scattering pattern such that a different object appears to be present. Despite the evident progress made in the development of passive wave-control methods, key challenges, such as the inherent loss due to frequency-dependent dissipation in the metamaterial, limited bandwidth, or their static time-invariant nature $[17,18]$, limit their practical applicability.

By contrast, active methods use externally controlled sources to provide energy to the acoustic system [19]. Recent advances in active metamaterials composed of periodic piezoelectric arrays offer new possibilities to change the effective material properties dynamically and therefore allow their functionality to adapt in real time $[20,21]$. In this connection, parity-time-symmetric materials have recently enabled material losses to be compensated [22]. The experimental realization of an invisible acoustic 
sensor in a one-dimensional (1D) waveguide based on a parity-time symmetric metamaterial device has been shown in Ref. [23]. The authors have demonstrated active cloaking of monochromatic wave fields while maintaining the wave field inside the cloaked region; however, cloaking could only be achieved in one direction due to the asymmetry of the system.

The idea of equipping a boundary with sources is closely related to the field of active noise control and antisound $[24,25]$. An active-noise-control experiment in which scattering from a three-dimensional (3D) object is reduced by an array of sources that emit adapted precomputed linear filters using Kirchhoff-Helmholtz integral expressions is presented in Ref. [26] (see also [27,28]). In these works, the scattered wave field is suppressed only after being scattered; thus transmissions into the scatterer are not controlled.

Also based on a Kirchhoff-Helmholtz integral formulation, the fact that perfect unidirectional cloaking can, in principle, be achieved when recording the waves near the surface of a scattering object and exciting appropriate point and dipole sources on the surface (assuming instantaneous calculation of the source strengths) has been shown in Ref. [29]. This approach is different from previous proposals as it manipulates the impinging waves rather than cancelling the scattered ones. Following up on this work, a method that significantly reduces the amount of surface sources by means of a multipole expansion has been proposed [30], though with the drawback of assuming full prior knowledge of the incident (monochromatic) field. Unfortunately, Refs. [29,30] give little insight into a practical implementation of the methods for the acoustic problem.

In this work, we use the concept of immersive boundary conditions (IBCs) to gain full control over an acoustic wave-propagation experiment. By applying a set of boundary conditions calculated in real time, reflections from boundaries of a physical domain can be suppressed. At the same time, and in contrast to previous methods, IBCs enable a dynamic coupling between the physical domain and a virtual (computational) environment $[31,32]$. In contrast to previous work, our method is not limited to monochromatic wave fields. Furthermore, we can choose between unidirectional or bidirectional operation, i.e., the virtual environment may exhibit different properties depending on the direction.

On the one hand, immersion can be facilitated to surround a physical domain by a virtual domain, thereby virtually extending the size of the physical domain. The first IBC experiment verifying the successful real-time extension of a physical-experimentation domain has been presented in Ref. [33] and, even more remarkably, has shown that long-range interactions between the physical and virtual domain can be accounted for. On the other hand, immersion also allows acoustic cloaking and holography [34]. In this case, a region inside the physical domain can be replaced by a virtual domain. As a result, scattering objects can be virtually substituted by arbitrary objects or by no objects at all.

We present an experimental demonstration of acoustic cloaking and holography using IBCs in a $1 \mathrm{D}$ waveguide. We review the concept of IBCs and introduce the experimental setup allowing for real-time implementation, including a feedback-loop between the physical and virtual domains. In a cloaking experiment, boundary conditions near a scattering object are manipulated so that the object effectively becomes invisible to a broadband wave field, without prior knowledge of the incident field. In a holography experiment, a scattering object acoustically appears where there is no object present. In a third experiment, an existing object is transformed such that the object exhibits properties that do not occur in nature. Lastly, we present an experiment with a directionally dependent virtual medium.

\section{THEORETICAL FRAMEWORK}

The theory of IBCs is introduced for the case of cloaking. A more general derivation can be found in Ref. [34]. Consider a scattering object that deflects an incident wave field propagating in a background medium [see Fig. 1(a)]. This is defined as the initial state, denoted below by subscript IN. The goal of IBCs is to substitute the presence of the physical scatterer by a medium equal to the background medium so that the scatterer is invisible for an observer outside of surface $S$ [see Fig. 1(c)]. This augmented state, denoted by subscript AG, is expressed as a combination of the initial state and a complementary state, denoted by IBC, such that the pressures are related by $p_{\mathrm{AG}}=p_{\mathrm{IN}}+p_{\mathrm{IBC}}$.

The pressure of the complementary state, $p_{\mathrm{IBC}}$, is derived from the acoustic representation theorem of the convolutional type $[34,35]$ and can be expressed as follows:

$$
\begin{aligned}
p_{\mathrm{AG}}(x, t)= & p_{\mathrm{IN}}(x, t) \\
& +\oint_{S}\left[G_{p, \mathrm{IN}}^{m}\left(x, x^{S}, t\right) * v_{i, \mathrm{AG}}\left(x^{S}, t\right)\right] n_{i} d S,
\end{aligned}
$$

with $x^{S} \in S$ and in which we assume that surface $S$ is rigid. $G_{p, \mathrm{IN}}^{m}\left(x, x^{S}, t\right)$ denotes the acoustic-pressure impulse response, or Green's function, of the initial state at location $x$ outside $S$ due to point sources of volume injection (monopoles) on $S . v_{i, \mathrm{AG}}\left(x^{S}, t\right)$ is the $i$ th component of the particle velocity in the augmented state, $n_{i}$ is the outwardpointing normal of $S$, and $*$ denotes convolution with respect to time. Equation (1) shows how a physical wave field can be manipulated to emulate propagation in the augmented state by applying specific boundary conditions on $S$. The boundary conditions must be interpreted as a distribution of monopole sources on $S$ with the strength of the particle velocity in the augmented state. Though not 


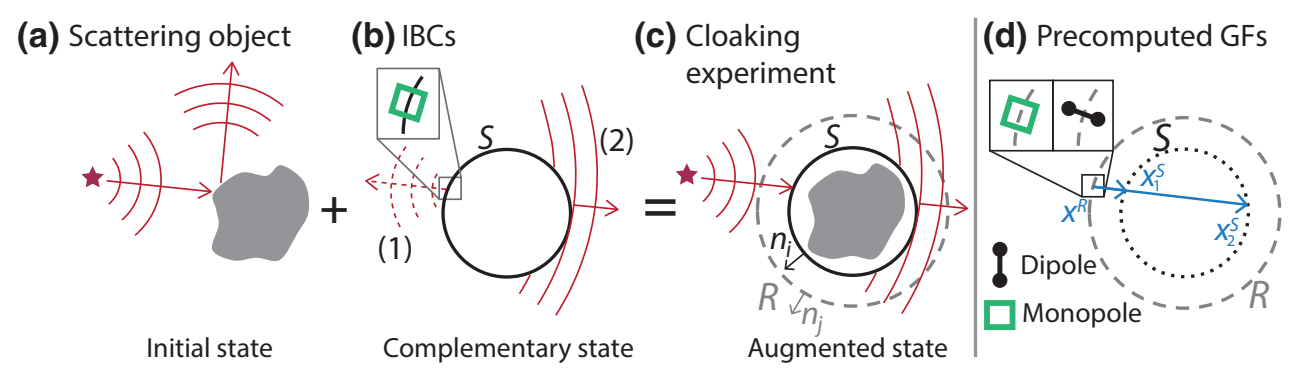

FIG. 1. The concept of IBCs for cloaking. (a) In the initial state, a wave field is perturbed by a scattering object. (b) A monopole source distribution on the rigid emitting surface $S$ cancels physical reflections of incoming waves (1) and emits calculated interactions with the virtual domain inside $S$ (2). (c) A cloaking experiment in which the IBCs virtually substitute the scatterer with the background medium. The signatures of the monopole sources on $S$ are derived by extrapolating wave-field recordings from a "sound-transparent" recording surface $R$ in real time. (d) The extrapolation step requires precomputed Green's functions (GFs) from each point on $R$ to each point on $S$ through the desired homogeneous virtual domain. Here, the Green's functions $G_{v_{i}, \mathrm{AG}}^{m, d_{j}}\left(x_{1}^{S}, x^{R}, t\right)$ and $G_{v_{i}, \mathrm{AG}}^{m, d_{j}}\left(x_{2}^{S}, x^{R}, t\right)$ carry the information to cancel incoming waves on $S$ and to re-emit transmitted waves in the "shadowed" part of the background medium, respectively.

further discussed herein, if $S$ was chosen to be a transparent boundary, the initial state inside $S$ could be maintained (assuming full knowledge of the medium inside $S$ ) [34]. Such a scenario could be advantageous, as it allows the design of invisible sensors. However, this concept is more challenging in practice, as it requires acoustically transparent monopole and dipole sources on $S$ as well as full knowledge of the medium inside $S$. For this reason, we focus on a rigid emitting surface.

To implement these boundary conditions in real time, the normal particle velocities $v_{i, \mathrm{AG}}\left(x^{S}, t\right)$ need to be known at the emitting surface $S$ before the wave physically arrives. Therefore, a transparent recording surface $R$ enclosing $S$ is introduced from which the recorded wave field must be extrapolated to $S$ [see Fig. 1(c)]. The extrapolation step is derived from the Kirchhoff-Helmholtz integral [36] as follows:

$$
\begin{aligned}
v_{i, \mathrm{AG}}\left(x^{S}, t\right)= & \oint_{R}\left[G_{v_{i}, \mathrm{AG}}^{d_{j}}\left(x^{S}, x^{R}, t\right) * p_{\mathrm{IN}}\left(x^{R}, t\right)\right. \\
& \left.+G_{v_{i}, \mathrm{AG}}^{m}\left(x^{S}, x^{R}, t\right) * v_{j, \mathrm{IN}}\left(x^{R}, t\right)\right] n_{j} d R,
\end{aligned}
$$

with $x^{R} \in R . G_{v_{i}, \mathrm{AG}}^{d_{j}}$ and $G_{v_{i}, \mathrm{AG}}^{m}$ are the velocity Green's functions recorded at $S$ due to a dipole $\left(d_{j}\right)$ and monopole (m) impulse at $R$, respectively. The Green's functions describe the desired wave propagation inside $R$ and are computed prior to the experiment - analytically, numerically, or experimentally. The key point is that the medium inside $S$ can be designed arbitrarily, allowing the real medium to be substituted by any desired medium (referred to as the virtual domain) from the point of view of an external observer. Figure 1(d) illustrates precomputation of the extrapolation Green's functions using the desired medium properties: e.g., for each point on a now sound-transparent surface $S$, the velocity impulse responses are calculated due to individual monopole and dipole sources on $R$ using a homogeneous background medium.

By combining measurements of the pressure and the normal particle velocity in Eq. (2), the wave field is implicitly separated into inward- and outward-propagating components at $R$ [37]. Thus, only components propagating inward from $R$ to $S$ are extrapolated, whereas components propagating outward do not contribute to $v_{i, \mathrm{AG}}\left(x^{S}, t\right)$. To

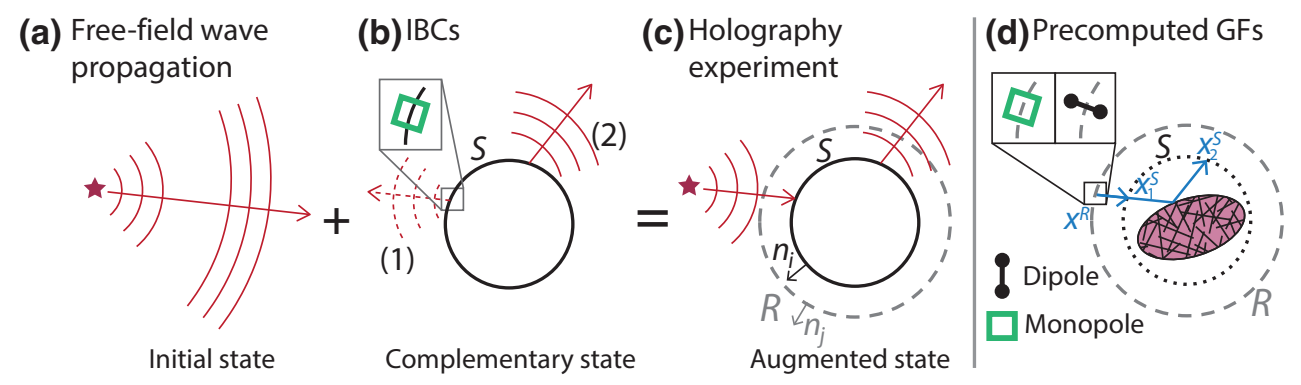

FIG. 2. The concept of IBCs for holography. (a) In the initial state, a wave field propagates unperturbed in a homogeneous medium. (b) Distribution of monopole sources on the rigid boundary $S$ effects cancellation of incoming waves on $S$ (1) and the emission of interaction with the virtual domain inside $S$ (2). (c) A holography experiment in which the IBCs virtually substitute the background medium with a scatterer. (d) The precomputation of GFs appropriate for the chosen scatterer. 
facilitate the numerical implementation of the extrapolation in real time, the temporal convolution in Eq. (2) is discretized in a time-recursive fashion and the surface integral is expressed as a matrix-vector multiplication such that the calculation can be performed recursively at each time step [31,33].

The concept of using IBCs to cloak an object can be extended to create acoustic illusions or holograms. Figure 2 depicts the setup for a holography experiment. In the initial state, a wave field propagates unperturbed in a homogeneous medium. The crucial difference to the cloaking experiment lies in the design of the virtual domain in Fig. 2(d). The extrapolation Green's functions can emulate propagation through arbitrarily complex objects (even phenomena that do not occur naturally).

\section{EXPERIMENTAL SETUP}

In the IBC experiments, air-filled tubes are used as waveguides to confine the wave propagation in one dimension. Ideally, only the fundamental first-order mode propagates unattenuated inside the tube when low frequencies are used with respect to the tube diameter $[38,39]$. Figure 3(a) shows two different geometries. In model A, the waveguide has a constant cross-section area producing a homogeneous 1D medium. In model B, the waveguide has a variable cross-section area: impedance contrasts at the discontinuities induce reflection and transmission of incident waves [38]. Hence, model $B$ represents a three-layered medium. For both models, physical reference experiments are carried out as shown in Fig. 3(b). Similar to the definition of the states in Fig. 1, model B is the initial state for the cloaking experiment and model A is the desired state; thus the IBCs virtually remove the impedance contrast. For the holography experiment, model A represents the initial state and model B is the desired state; thus the IBCs create a virtual impedance-contrast layer. For the third experiment, in which the physics of an existing object is modified by the IBCs, the initial state is represented by model $\mathrm{B}$, whereas the desired state is represented by a numerical model exhibiting the same (layered) geometry but the middle layer reveals properties of an "energy-gain" medium. Table I summarizes the initial and desired states for the cloaking and holography experiments.

The experimental IBC setup is sketched in Fig. 3(c), in which the physical domain $D_{\text {phys }}$ is interrupted by the virtual domain $D_{\text {virt }}$. A loudspeaker mounted on the left end of the left tube excites an arbitrary wave field and a movable microphone records the pressure field at multiple locations inside the tubes (denoted "stim" and "ref mic," respectively). The end of the right tube is terminated by a rigid boundary. The IBC ingredients can be classified into three parts: the emitting surface, the recording surface and the data-acquisition and control system. The discrete emitting
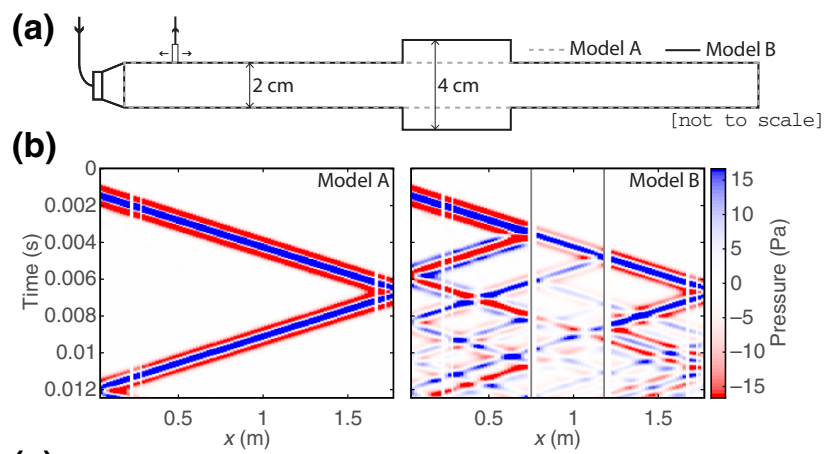

(c)

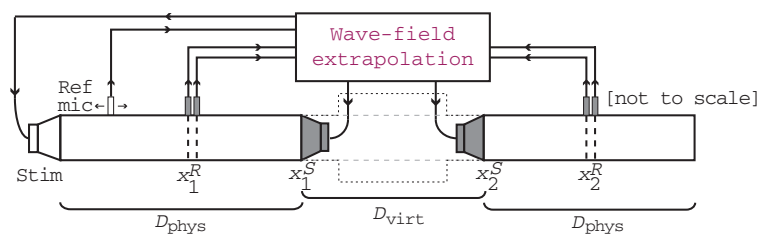

(d)

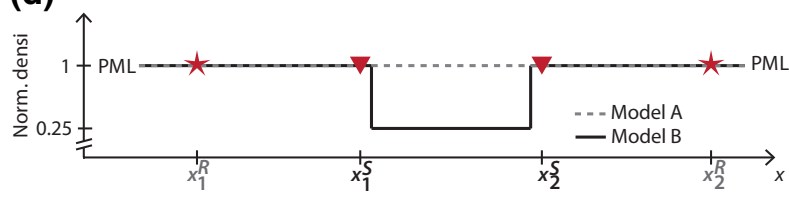

FIG. 3. (a) A schematic of the reference experiments: a waveguide with a constant cross-section area (model A, dashed line) and a waveguide with a layer of increased cross-section area (model B, solid line). (b) Pressure data from the reference experiments. (c) A schematic of the experimental setup. (d) The normalized density model used to numerically compute the Green's functions $G\left(x_{1,2}^{S}, x_{1,2}^{R}\right)$ prior to the IBC experiment. The two stars indicate positions at which a delta impulse is injected in two successive simulations and triangles indicate positions at which the propagated delta impulse is recorded. The model is surrounded by PML layers to absorb outgoing waves [40].

surface consists of two loudspeakers at positions $x_{1,2}^{S}$ which enclose the virtual domain. The recording positions are located in the outward direction of the emitting positions and are denoted by $x_{1,2}^{R}$. Equation (2) shows that both the pressure and the particle-velocity recordings are required to enable the implicit wave-field separation. However, here the particle velocity may be derived by finite differencing the pressure at two closely spaced microphones at each recording position. This calculation is incorporated into the real-time extrapolation step by manipulating the Green's

TABLE I. The definition of the initial and desired states for the cloaking and holography experiments.

\begin{tabular}{lcc}
\hline \hline IBC experiment & Initial state & Desired state \\
\hline Cloaking & Model B & Model A \\
Holography & Model A & Model B \\
Holography (gain medium) & Model B & Nonphysical \\
Bidirectional & & $x<x_{1}^{S}$ : model A \\
& & $x>x_{2}^{S}$ : model B \\
\hline \hline
\end{tabular}



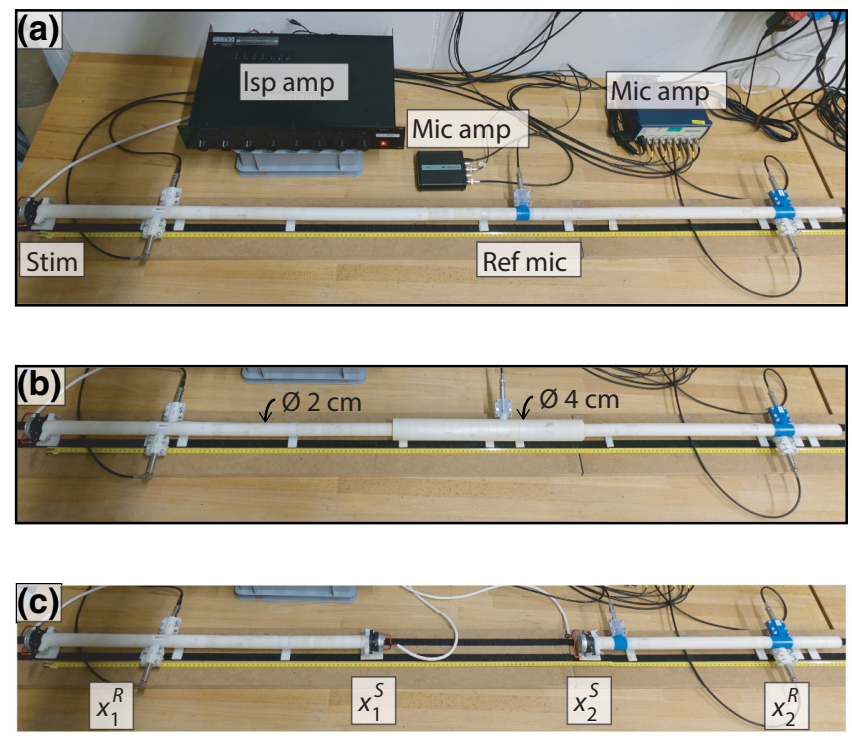

FIG. 4. The experimental setup. The waveguides are composed of 3D-printed 2-cm-long segments to be able to record the wave field at multiple positions inside the waveguide. All loudspeakers are amplified through a power amplifier ("lsp amp") and all analog microphone data are preamplified through a signal conditioner ("mic amp"). (a) The constant-diameter waveguide represents model A. A movable reference microphone ("ref mic") records the pressure inside the waveguide at multiple positions. (b) The variable-diameter waveguide represents model B. A change of the diameter from $2 \mathrm{~cm}$ to $4 \mathrm{~cm}$ produces reflection and transmission coefficients of $R=-0.6$ and $T=0.64$, respectively. In both reference models, the microphones at the sound-transparent recording positions are only used as additional (static) reference microphones. (c) The IBC experiment: at each recording position $\left(x_{1}^{R}\right.$ and $\left.x_{2}^{R}\right)$, two microphones separated by $1.8 \mathrm{~cm}$ are installed to measure the pressure and its gradient. Loudspeakers are mounted on the emitting boundary $\left(x_{1}^{S}\right.$ and $\left.x_{2}^{S}\right)$. The data-acquisition and control system is not shown here but is discussed in detail in Ref. [33].

functions appropriately [33]. The experimental setup is shown in Fig. 4.

The extrapolation Green's functions in Eq. (2) are precomputed numerically using a $1 \mathrm{D}$ second-order accurate finite-difference scheme in the time domain, solving the first-order representation of the acoustic wave equation [41]. The increased cross-section area in model B is implemented as an inversely proportional change in impedance in the numerical model [38]. For example, if the diameter of the waveguide changes from $d_{0}$ to $d_{1}=a d_{0}$, the density in the numerical model changes from $\rho_{0}$ to $\rho_{1}=$ $(1+b) /(1-b) \rho_{0}$ with $b=\left(1-a^{2}\right) /\left(1+a^{2}\right)$ while the velocity remains constant. Figure 3(d) shows the 1D density model used to compute the Green's functions representing models $\mathrm{A}$ and $\mathrm{B}$. The model is surrounded by perfectly matched layers (PMLs) to simulate an effectively infinite medium. As mentioned in Sec. II, the model can be designed arbitrarily outside $R$. Though ideally the fundamental mode is expected to propagate unattenuated in the waveguide, we observe an exponential amplitude decay of the wave with the propagation distance. The sections with diameters of $2 \mathrm{~cm}$ and $4 \mathrm{~cm}$ reveal a decay of $-2.76 \mathrm{~dB} \mathrm{~m}^{-1}$ and $-1.01 \mathrm{~dB} \mathrm{~m}^{-1}$, respectively. To accurately account for this, we use a damped acoustic wave equation in the finite-difference scheme [42]. While the attenuation is frequency independent within the frequency band of our experiments, it is a function of the tube diameter. Appropriate damping coefficients for the numerical model are found by matching experimental and numerical data.

A high-performance data-acquisition and control system carries out the extrapolation in real time, so that the computation is faster than the physical propagation of the wave from $R$ to $S$. The system operates at a sampling frequency of $20 \mathrm{kHz}$ and guarantees a latency of $0.2 \mathrm{~ms}$ (four time samples), including the collection of all receiver data, execution of the matrix-vector multiplication, and emission of the extrapolated quantities at all emitting positions. In addition to the system latency, corrections minimizing undesired hardware effects contribute significantly to the total latency. These undesired effects include the frequency-dependent loudspeaker transfer function and the fact that the emitting surfaces (the loudspeakers) are not perfectly rigid [33]. The total latency dictates the separation distance between $R$ and $S$. Here, a distance of $0.45 \mathrm{~m}$ is needed to allow for an extrapolation time of $1.31 \mathrm{~ms}$, including all hardware corrections.

\section{RESULTS AND DISCUSSION}

In the cloaking experiment, extrapolation Green's functions represent a homogeneous virtual domain (model A). The Green's functions, $G\left(x_{1}^{S}, x_{1}^{R}, t\right)$ and $G\left(x_{2}^{S}, x_{2}^{R}, t\right)$, suppress boundary reflections from incoming waves so that the loudspeaker boundary appears acoustically transparent from outside $S$. In addition, $G\left(x_{1}^{S}, x_{2}^{R}, t\right)$ and $G\left(x_{2}^{S}, x_{1}^{R}, t\right)$ account for the forward propagation of the incoming waves through the virtual domain. The experimental results are displayed in Fig. 5. The convincing match between the desired state (the reference data of model A) and the IBC experiment shows that the IBCs allow us to fully embed an arbitrary virtual medium into a physical experiment and to cloak any physical medium that is collocated with the virtual medium. Some spurious energy due to the imperfect suppression of the reflection at $S$ can be observed [e.g., IBC data in the top panel of Fig. 5(b), for $0.005 \mathrm{~s}<t<$ $0.007 \mathrm{~s}]$. This residual energy is likely a result of imperfect correction for the transfer function of the loudspeaker.

Figure 6 gives an example of the suppression of the boundary reflection occurring at $x_{1}^{S}$. Pressure data recorded at location $x=0.37 \mathrm{~m}$ from a cloaking experiment with IBCs turned on (dotted line) are compared to an experiment with IBCs turned off (solid line). The 


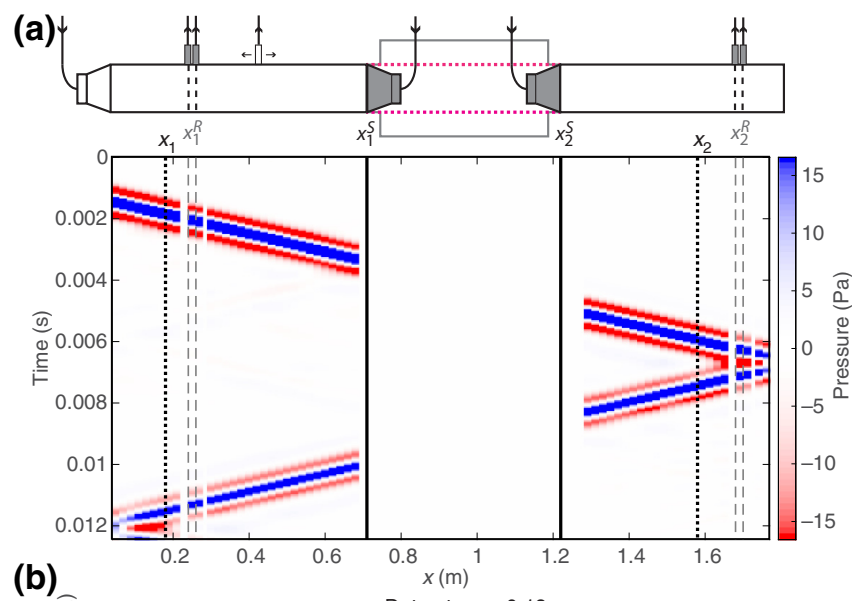

(b)

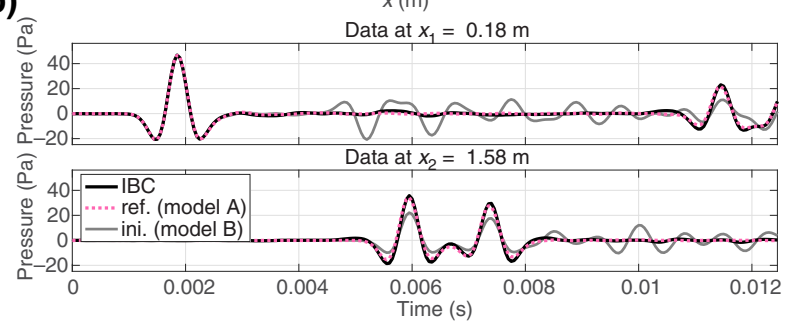

FIG. 5. The IBC cloaking experiment with Green's functions representing a homogeneous virtual domain. A Ricker wavelet with a central frequency of $1 \mathrm{kHz}$ is emitted by the left loudspeaker. (a) The time-distance panel. (b) Pressure recordings of the IBC experiment at $x_{1}$ and $x_{2}$ are compared to their initial state and a reference experiment (the desired state).

time window $t_{a}<t<t_{b}$ contains the temporally isolated reflected wave (and the suppressed reflection). Calculation of the acoustic intensity $\left[\sum_{t=t_{a}}^{t_{b}} p^{2}(x, t)\right]$ ratio between
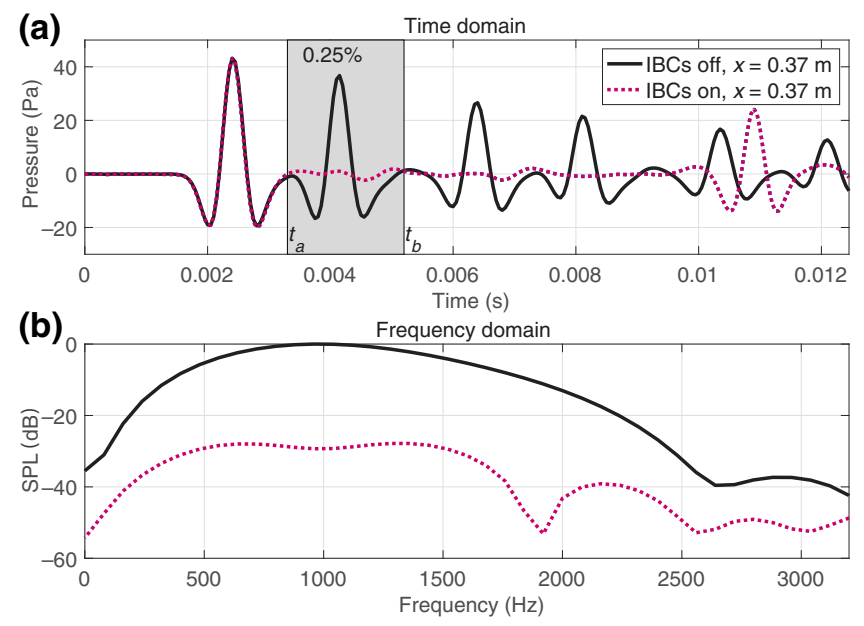

FIG. 6. A comparison of cloaking experiments with IBCs turned on (dotted line) and IBCs turned off (solid line) to evaluate the suppression of the reflection at the emitting boundary. (a) The time-domain signals recorded at location $x=0.37 \mathrm{~m}$. (b) The sound-pressure level as a function of the frequency for the time window $t_{a}<t<t_{b}$. the two experiments for the above time window shows that the reflection is suppressed down to $0.25 \%$. A Fourier transform of the windowed pressure data shows that the sound-pressure level (SPL) is reduced by approximately 20 $\mathrm{dB}$ over a broad frequency range when IBCs are turned on. The remaining energy is mainly due to the imperfect correction for the frequency-dependent loudspeaker transfer function. The correction is implemented as a deconvolution of the measured transfer function (in the frequency range of our experiment) from the Green's functions, which can be carried out prior to the experiment [33]. Note that further improvements in the correction come at the expense of increasing the latency to compute the extrapolation step, therefore increasing the separation between $S$ and $R$.

The real-time wave-field separation becomes apparent at the recording surface $x_{2}^{R}$, where right- and left-going waves overlap due to the close vicinity of the rigid termination (Fig. 5). As explained in Sec. II, the Green's functions are designed to extrapolate only waves propagating in the direction from $x^{R}$ to $x^{S}$. This means that at $x_{2}^{R}$, only the leftgoing wave must be extrapolated, which is ensured by the real-time wave-field separation [33].

The reflection at the right-side rigid termination acts as a secondary source, showing that the cloaking is unidirectional and works for any number of sources located outside the recording surface.

In the holography experiment, the extrapolation Green's functions represent a more complex virtual domain [model $B$ in Fig. 3(a)]. In addition to the cancellation and transmission events, the Green's functions now include

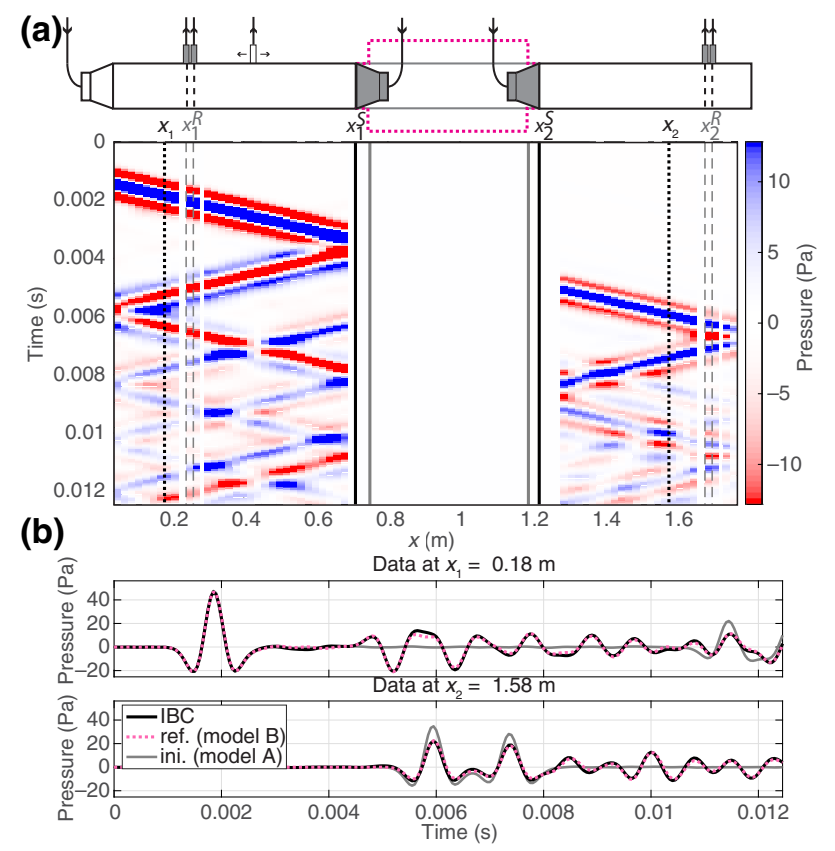

FIG. 7. The IBC holography experiment, with Green's functions representing a heterogeneous virtual domain. 
all internal multiples caused by the virtual impedance contrasts. Figure 7 displays the results of the IBC experiment together with the reference experiments. Here, longrange interactions between the physical and virtual domain become rather complex. The comparison with a reference experiment in Fig. 3(b) is therefore helpful to benchmark that the IBCs are implemented correctly. Clearly, the geometry and physics of the virtual domain are indeed captured as intended by the extrapolation Green's functions.

So far, we have used the IBCs to reconstruct physically possible virtual domains. We now augment the physical domain by a virtual domain that does not occur in nature. The section of lower impedance (larger diameter) in the virtual domain is designed as an "energy-gain" medium in the numerical model. Figure 8(a) (middle panel) shows the desired amplitude behavior of a right-going wave and a left-going wave reflected at the second impedance contrast: the attenuation in the layers of smaller diameter is equivalent to the experimentally observed attenuation in

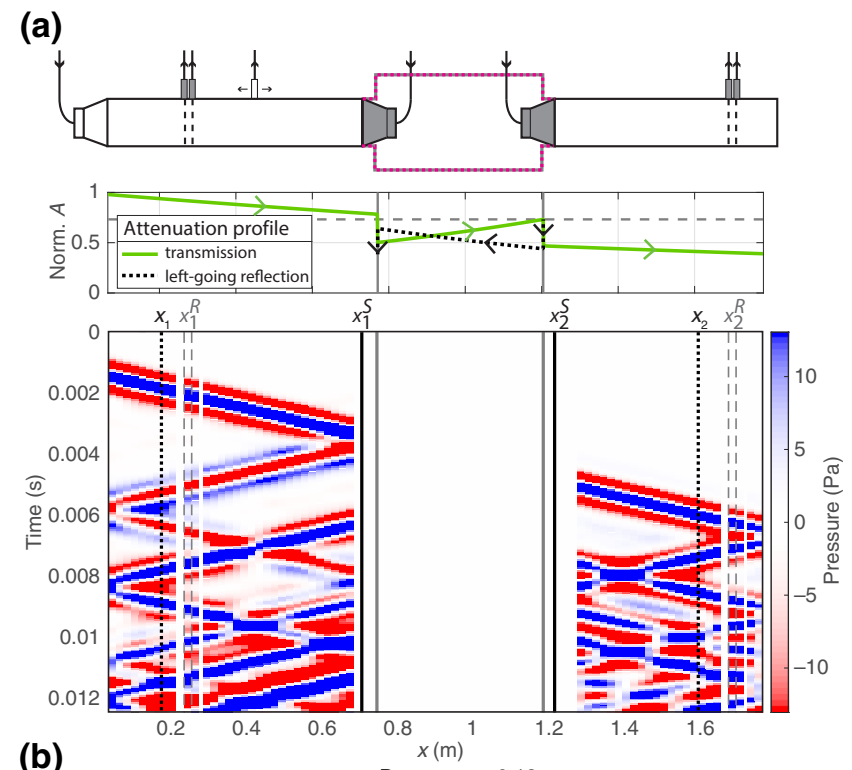

(b)

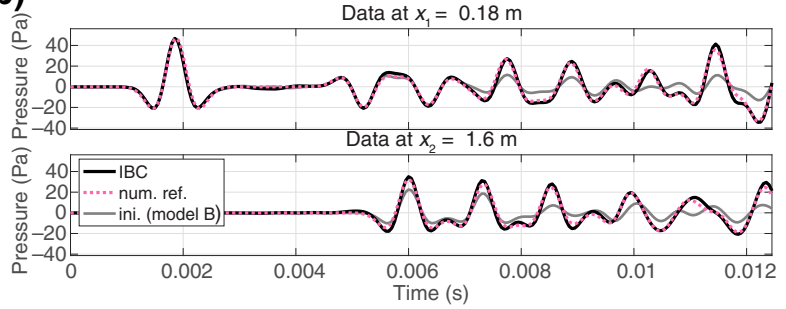

FIG. 8. The IBC experiment with Green's functions representing a heterogeneous virtual domain containing an "energy-gain" medium. The middle panel in (a) shows an attenuation profile of the desired state for a left-going transmission and a reflection at the second interface. When numerically calculating the Green's functions for the IBC experiment, the lower-diameter layers exhibit an attenuation identical to the experimentally observed attenuation. By contrast, the larger-diameter layer is designed such that it reveals "energy-gain" properties [42]. the waveguide, i.e., $-2.76 \mathrm{~dB} / \mathrm{m}$. By contrast, we assign a gain of $7.57 \mathrm{~dB} / \mathrm{m}$ to the middle layer of larger diameter. The gain over the length of the middle layer does not exceed the loss due to the transmission at the discontinuities, from which it follows that internal multiples do not intensify with each reverberation. Nonetheless, the gain inside the virtual domain leads to increased amplitudes in the physical domain, as can be seen in the lower panel of Fig. 8(a). Due to the impossibility of a physical reference experiment including a gain medium, the IBC experiment is compared to the numerical simulation in Fig. 8(b).

The concept can be extended further to create virtual media with directionally dependent properties. These have similarities with parity-time-symmetric experiments $[23,43]$. In Fig. 9, we show how the Green's functions can be manipulated to emulate a virtual medium that appears homogeneous for an observer at $x<x_{1}^{S}$ and heterogeneous for an observer at $x>x_{2}^{S}$. Note that due to the attenuation associated with the transmission through the heterogeneous virtual domain (i.e., from $x_{1}^{R}$ to $x_{2}^{S}$ ),

(a)
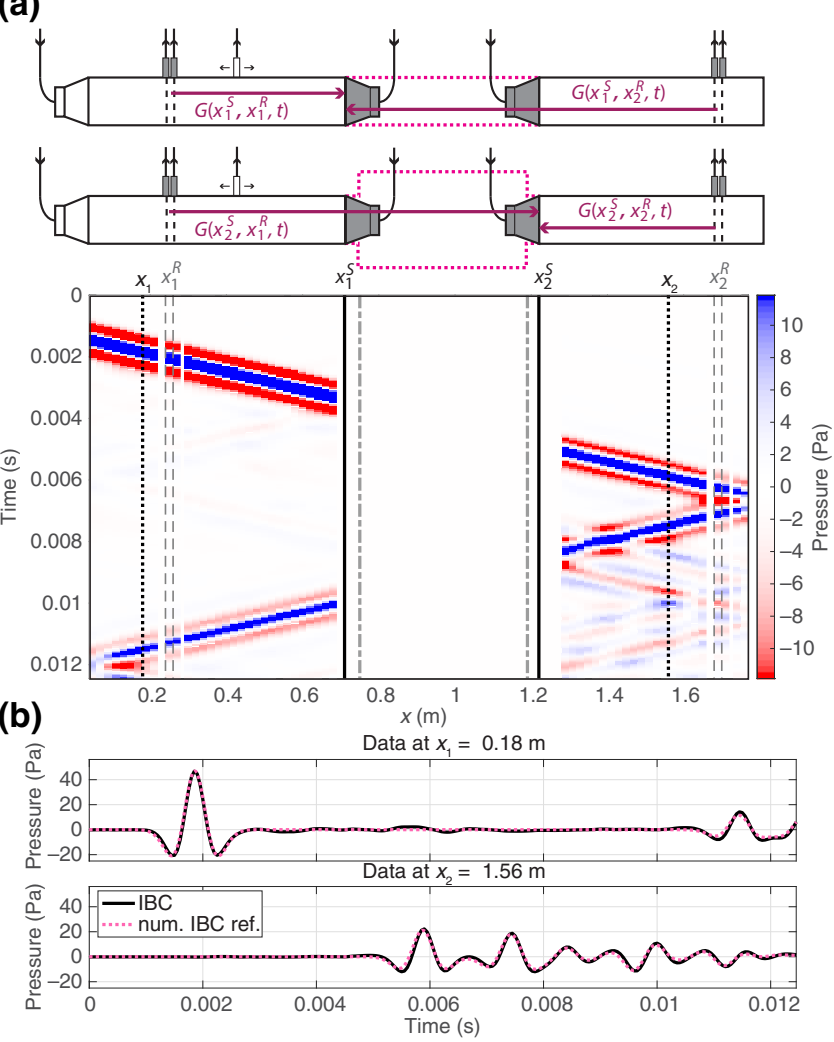

FIG. 9. The IBC experiment with Green's functions representing a homogeneous and heterogeneous virtual domain simultaneously, depending on the location of the observer. (a) While Green's functions $G\left(x_{1}^{S}, x_{1}^{R}\right)$ and $G\left(x_{1}^{S}, x_{2}^{R}\right)$ emulate a homogeneous virtual domain as in the cloaking experiment, $G\left(x_{2}^{S}, x_{1}^{R}\right)$ and $G\left(x_{2}^{S}, x_{2}^{R}\right)$ emulate a heterogeneous virtual domain as in the holography experiment. (b) A comparison with a numerical IBC simulation in which the above Green's functions are used. 
this experiment is different from "stitching" together the results of the cloaking and holography experiments for $x<$ $x_{1}^{S}$ and $x>x_{2}^{S}$, respectively. However, attenuation losses could be compensated for, as shown by the previous experiment.

The method of IBCs is not restricted to air. In fact, the medium inside $S^{\mathrm{emt}}$ and outside $S^{\mathrm{rec}}$ can be chosen arbitrarily. The only requirement to facilitate a real-time extrapolation of the wave field is that the medium between $S^{\mathrm{emt}}$ and $S^{\mathrm{rec}}$ needs to be a fluid. For example, an underwater application with the data-acquisition and control system used in this study is deployable under the condition that the distance between the recording and emitting surfaces is adapted according to the high acoustic velocity of water and the total latency of the system (including hardware effects). However, due to the high acoustic impedance of water, a 1D or two-dimensional (2D) waveguide would be impractical to implement and therefore a 3D setup would be required.

For 2D and 3D IBC experiments, additional challenges emerge: spatial subsampling associated with discretization of the recording and emitting surfaces, or the difficulty of realizing an acoustically transparent recording device. Nonetheless, our experimental results validate the concept for implementing IBCs in 2D and 3D laboratories.

An extension to elastic media with IBCs implemented on a free surface of an elastic (solid) object is presented in Ref. [44]. Since the wave field cannot be accessed nondestructively inside the object, the recording surface coincides with the emitting surface (on the free surface), so that energy arriving at the source boundary cannot be predicted in real time and IBCs must be constructed time step by time step as waves are recorded. The central challenge of elastic IBCs is to separate the wave field recorded at the free surface into its incident and reflected components, which can be achieved by using an elastic finite-difference wave-field injection technique.

Our method can also be applied to the field of electromagnetic wave physics, though implementation in real time may be unrealistic due to the high propagation velocity of electromagnetic waves. Even for an underwater application, where the speed of electromagnetic waves is significantly lower than in air, a real-time extrapolation would require an extremely low-latency data-acquisition and control system.

\section{CONCLUSION}

We demonstrate that IBCs enable full control over broadband wave fields and can be used to augment a physical experiment with a virtual domain in real time. The immersion is achieved by calculating instead of physically propagating a wave field through an arbitrary virtual domain. At the same time, scattering from the boundary of the virtual domain is compensated for. We immerse the physical domain into three different scenarios. First, we control the wave field of a scattering medium as though propagation was through a homogeneous medium (the cloaking experiment). Second, we show that long-range interactions between the physical and a heterogeneous virtual domain are fully accounted for (the holography experiment). Third, we emulate a nonphysical virtual domain with properties of an "energy-gain" medium.

In future work, we envisage simulating time-variant media in the virtual domain. Furthermore, the physical realization of higher-dimensional experiments is work in progress.

\section{ACKNOWLEDGMENTS}

This work was funded by the European Union's Horizon 2020 Research and Innovation Programme under the Marie Sklodowska-Curie Grant Agreement No. 641943. Furthermore, this project has received funding from the European Research Council (ERC) under the European Union's Horizon 2020 Research and Innovation Programme (Grant Agreement No. 694407).

[1] D. Schurig, J. Mock, B. Justice, S. A. Cummer, J. B. Pendry, A. Starr, and D. Smith, Metamaterial electromagnetic cloak at microwave frequencies, Science 314, 977 (2006).

[2] J. Valentine, S. Zhang, T. Zentgraf, E. Ulin-Avila, D. A. Genov, G. Bartal, and X. Zhang, Three-dimensional optical metamaterial with a negative refractive index, Nature $\mathbf{4 5 5}$, 376 (2008).

[3] R. V. Craster and S. Guenneau, Acoustic Metamaterials: Negative Refraction, Imaging, Lensing and Cloaking (Springer Science \& Business Media, Dordrecht, 2013).

[4] G. Koutromanos, A. Sofos, and L. Avraamidou, The use of augmented reality games in education: A review of the literature, EMI Educ. Media Int. 52, 253 (2015).

[5] B. H. Thomas, A survey of visual, mixed, and augmented reality gaming, Comput. Entertainment 10, 3 (2012).

[6] J. B. Pendry, Negative Refraction Makes a Perfect Lens, Phys. Rev. Lett. 85, 3966 (2000).

[7] D. R. Smith, D. Vier, N. Kroll, and S. Schultz, Direct calculation of permeability and permittivity for a left-handed metamaterial, Appl. Phys. Lett. 77, 2246 (2000).

[8] W. Cai and V. Shalaev, Optical Metamaterials: Fundamentals and Applications (Springer Science \& Business Media, New York, 2010).

[9] A. B. Movchan and S. Guenneau, Split-ring resonators and localized modes, Phys. Rev. B 70, 125116 (2004).

[10] T. R. Howarth, V. K. Varadan, X. Bao, and V. V. Varadan, Piezocomposite coating for active underwater sound reduction, J. Acoust. Soc. Am. 91, 823 (1992).

[11] B. I. Popa, L. Zigoneanu, and S. A. Cummer, Experimental Acoustic Ground Cloak in Air, Phys. Rev. Lett. 106, 253901 (2011).

[12] L. Zigoneanu, B.-I. Popa, and S. A. Cummer, Threedimensional broadband omnidirectional acoustic ground cloak, Nat. Mater. 13, 352 (2014). 
[13] A. Colombi, P. Roux, S. Guenneau, P. Gueguen, and R. V. Craster, Forests as a natural seismic metamaterial: Rayleigh wave bandgaps induced by local resonances, Sci. Rep. 6, 19238 (2016).

[14] P. Kerrian, A. Hanford, B. Beck, and D. Capone, Underwater acoustic ground cloak development and demonstration, J. Acoust. Soc. Am. 143, 1918 (2018).

[15] W. Kan, B. Liang, X. Zhu, R. Li, X. Zou, H. Wu, J. Yang, and J. Cheng, Acoustic illusion near boundaries of arbitrary curved geometry, Sci. Rep. 3, 1427 (2013).

[16] Y. Liu and S. He, Acoustic Illusion Using Materials with Isotropic and Positive Parameters, Phys. Rev. Appl. 10, 064036 (2018).

[17] F. Monticone and A. Alù, Do Cloaked Objects Really Scatter Less? Phys. Rev. X 3, 041005 (2013).

[18] R. Fleury, F. Monticone, and A. Alù, Invisibility and Cloaking: Origins, Present, and Future Perspectives, Phys. Rev. Appl. 4, 037001 (2015).

[19] S. A. Cummer, J. Christensen, and A. Alù, Controlling sound with acoustic metamaterials, Nat. Rev. Mater. 1, 16001 (2016).

[20] W. Akl and A. Baz, Analysis and experimental demonstration of an active acoustic metamaterial cell, J. Appl. Phys. 111, 044505 (2012).

[21] B. I. Popa, D. Shinde, A. Konneker, and S. A. Cummer, Active acoustic metamaterials reconfigurable in real time, Phys. Rev. B 91, 220303 (2015).

[22] R. Fleury, D. L. Sounas, and A. Alù, Parity-time symmetry in acoustics: Theory, devices, and potential applications, IEEE J. Quantum Electron. 22, 121 (2016).

[23] R. Fleury, D. Sounas, and A. Alù, An invisible acoustic sensor based on parity-time symmetry, Nat. Commun. 6, 5905 (2015).

[24] J. F. Williams, Review lecture: Anti-sound, Proc. R. Soc. London Sect. A 395, 63 (1984).

[25] S. J. Elliott and P. A. Nelson, Active noise control, IEEE Signal Process. Mag. 10, 12 (1993).

[26] E. Friot, R. Guillermin, and M. Winninger, Active control of scattered acoustic radiation: A real-time implementation for a three-dimensional object, Acta Acust. United Acust. 92, 278 (2006).

[27] S. Ise, A principle of sound field control based on the Kirchhoff-Helmholtz integral equation and the theory of inverse systems, Acta Acust. United Acust. 85, 78 (1999).

[28] S. Uosukainen, Active sound scatterers based on the JMC method, J. Sound Vib. 267, 979 (2003).

[29] D. A. Miller, On perfect cloaking, Opt. Express 14, 12457 (2006).
[30] F. G. Vasquez, G. W. Milton, and D. Onofrei, Exterior cloaking with active sources in two dimensional acoustics, Wave Motion 48, 515 (2011).

[31] D.-J. van Manen, J. O. Robertsson, and A. Curtis, Exact wave field simulation for finite-volume scattering problems, J. Acoust. Soc. Am. 122, EL115 (2007).

[32] M. Vasmel, J. O. Robertsson, D.-J. van Manen, and A. Curtis, Immersive experimentation in a wave propagation laboratory, J. Acoust. Soc. Am. 134, EL492 (2013).

[33] T. S. Becker, D.-J. van Manen, C. M. Donahue, C. Bärlocher, N. Börsing, F. Broggini, T. Haag, J. O. A. Robertsson, D. R. Schmidt, S. A. Greenhalgh, and T. E. Blum, Immersive Wave Propagation Experimentation: Physical Implementation and One-Dimensional Acoustic Results, Phys. Rev. X 8, 031011 (2018).

[34] D.-J. van Manen, M. Vasmel, S. Greenhalgh, and J. O. A. Robertsson, Broadband cloaking and holography with exact boundary conditions, J. Acoust. Soc. Am. 137, EL415 (2015).

[35] J. T. Fokkema and P. M. van denBerg, Seismic Applications of Acoustic Reciprocity (Elsevier, Amsterdam, 1993).

[36] A. J. Berkhout, Applied Seismic Wave Theory (Elsevier, New York, 1987).

[37] J. F. Claerbout, Toward a unified theory of reflector mapping, Geophysics 36, 467 (1971).

[38] F. Jacobsen, Propagation of sound waves in ducts, Note No. 31260 (Technical University of Denmark, Lyngby, 2008).

[39] P. M. Morse and K. U. Ingard, Theoretical Acoustics (McGraw Hill, New York, 1968).

[40] D. Komatitsch and R Martin, An unsplit convolutional perfectly matched layer improved at grazing incidence for the seismic wave equation, Geophysics 72, SM155 (1997).

[41] J. Virieux, P-SV wave propagation in heterogeneous media: Velocity-stress finite-difference method, Geophysics 51, 889 (1986).

[42] Q.-H. Liu and J. Tao, The perfectly matched layer for acoustic waves in absorptive media, J. Acoust. Soc. Am. 102, 2072 (1997).

[43] H.-X. Li, M. Rosendo-Lopez, Y.-F. Zhu, X.-D. Fan, D. Torrent, B. Liang, J.-C. Cheng, and J. Christensen, Ultrathin acoustic parity-time symmetric metasurface cloak, arXiv:1812.05845 (2018).

[44] H. R. Thomsen, D.-J. van Manen, and J. O. A. Robertsson, Exact wavefield separation on an elastic-free surface with sharp corners, SEG Technical Program Expanded Abstracts (2018), p. 5017. 